\title{
Attenuation of sepsis-induced rat liver injury by epigallocatechin gallate via suppression of oxidative stress-related inflammation
}

\author{
Jian-xin Yang ${ }^{1}$, Yu-lin Li ${ }^{1 *}$, Ning-chuan Shi ${ }^{2}$ \\ ${ }^{1}$ Department of Emergency, The Second Affiliated Hospital, College of Medicine, Zhejiang University, Hangzhou, Zhejiang, \\ 310009, ${ }^{2}$ College of Life Sciences, Zhejiang Chinese Medical University, Hangzhou, Zhejiang, 310053, PR China
}

*For correspondence: Email: 2513003@zju.edu.cn

\begin{abstract}
Purpose: To evaluate the effect of epigallocatechin gallate (EGCG) on sepsis-induced liver injury in a rat model of sepsis established by cercal ligation and puncture (CLP).

Methods: Male Wistar rats were randomly divided into 6 groups $(n=12)$ : normal control, sepsis, dexamethasone (5 mg/kg), low-dose EGCG (12.5 mg/kg), medium-dose EGCG $(25 \mathrm{mg} / \mathrm{kg})$, and highdose EGCG $(50 \mathrm{mg} / \mathrm{kg})$ groups. Dexamethasone and EGCG were given once daily. Survival rates following CLP were recorded. Serum alanine aminotransferase (ALT) and aspartate aminotransferase (AST) levels were measured to evaluate liver function. Tumor necrosis factor- $\alpha$ (TNF- $\alpha$ ) and interleukin (IL)-10 were determined by ELISA. Superoxide dismutase (SOD) and levels of glutathione (GSH) and malondialdehyde (MDA) were assayed to evaluate oxidative stress. Protein and mRNA expression levels of heme oxygenase-1 (HO-1) and nuclear factor erythroid 2-related factor (Nrf2) were measured by Western blotting and quantitative real-time polymerase chain reaction ( $q R T-P C R)$, respectively.

Results: Survival rates were significantly $(p<0.05)$ increased by EGCG $(83.3 \%)$ when compared to the untreated sepsis group (33.3 \%) or dexamethasone-treated sepsis group (41.7\%). The increase in survival rates was associated with significant decreases in AST, ALT, MDA, and TNF-a, and significant elevations in SOD, GSH, and IL-10. QRT-PCR and Western blotting data indicate that there was increase in hepatic expression of Nrf-2 and HO-1 of EGCG-treated sepsis rats, relative to the untreated sepsis group.

Conclusion: These results suggest that EGCG treatment reduces sepsis-induced liver injury and improves the survival rate of rats with polymicrobial sepsis by reducing oxidative stress via regulation of $\mathrm{Nrf2/HO-1} \mathrm{signaling.} \mathrm{These} \mathrm{findings} \mathrm{highlight} \mathrm{the} \mathrm{promising} \mathrm{potential} \mathrm{of} \mathrm{EGCG} \mathrm{for} \mathrm{the} \mathrm{treatment} \mathrm{of}$ sepsis.
\end{abstract}

Keywords: Epigallocatechin gallate, Cecal ligation and puncture (CLP), Sepsis, Liver injury, Oxidative stress, Inflammation

This is an Open Access article that uses a funding model which does not charge readers or their institutions for access and distributed under the terms of the Creative Commons Attribution License (http://creativecommons.org/licenses/by/4.0) and the Budapest Open Access Initiative (http://www.budapestopenaccessinitiative.org/read), which permit unrestricted use, distribution, and reproduction in any medium, provided the original work is properly credited.

Tropical Journal of Pharmaceutical Research is indexed by Science Citation Index (SciSearch), Scopus, International Pharmaceutical Abstract, Chemical Abstracts, Embase, Index Copernicus, EBSCO, African Index Medicus, JournalSeek, Journal Citation Reports/Science Edition, Directory of Open Access Journals (DOAJ), African Journal Online, Bioline International, Open-J-Gate and Pharmacy Abstracts

\section{INTRODUCTION}

Sepsis is often the main cause of death in a surgical intensive care unit (ICU), and is still a worldwide challenge [1]. Multiple proinflammatory cytokines (including TNF- $\alpha$ are accumulated during sepsis and are involved in the pathogenesis of sepsis [2]. 
Systemic inflammation could lead to multiple organ injury, including liver injury. Liver injury in sepsis progresses from active hepatocellular dysfunction to liver damage and finally to liver failure [3]. The liver performs a wide range of biological functions in metabolic and immunological homeostasis, and contributes to bacterial clearance in sepsis [4]. It has been demonstrated that liver failure is significantly associated with poor prognosis in critically ill sepsis patients [5].

Oxidative stress occurs when the rate of reactive species generation exceeds the antioxidant capacity, resulting in excessive oxidants in cells [6]. Accumulating evidence demonstrate that severe oxidative stress is present in patients with sepsis, due to overload of reactive oxygen species (ROS) and inadequate anti-oxidative defenses [7]. Oxidative stress is involved in the pathology of sepsis through several different mechanisms, and it is highly associated with inflammation [8]. Oxidative stress directly attacks endothelial cells and affects oxygen consumption, thus promoting organ failure [9]. Therefore, in sepsis situations, it is important to overcome oxidative stress and the resulting disease progression using antioxidants.

Green tea contains four major polyphenols, of which EGCG is the most active antioxidant component [10]. In the present study, the protective role of EGCG against oxidative stress and liver injury in rats subjected to CLP-induced sepsis was investigated.

\section{EXPERIMENTAL}

\section{Animals and reagents}

Adult male Wistar rats (mean body weight $=250$ $\pm 10 \mathrm{~g}$ ) were housed three per metabolic cage under a $12 \mathrm{~h}$ light-12 h dark cycle. All rats were maintained for seven days to observe their health condition before the experiment. All the animal experiments were approved by the Animal Care and Use Committee of the Second Affiliated Hospital of Zhejiang University School of Medicine (ref no. 2016-13), and were in accordance with Principles of Laboratory Animal Care (NIH publication no. 85-23, revised 1985) [11]. Dexamethasone and EGCG were obtained from Sigma (St. Louis, USA).

The adult male Wistar rats were divided into six groups ( $n=12$ in each group): normal control group, sepsis group (septic rats without treatment), dexamethasone group (septic rats treated with $5 \mathrm{mg} / \mathrm{kg}$ dexamethasone), low-dose
EGCG group (septic rats treated with $12.5 \mathrm{mg} / \mathrm{kg}$ EGCG), medium-dose EGCG group (septic rats

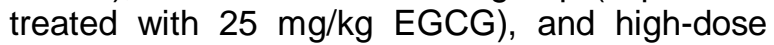
EGCG group (septic rats treated with $50 \mathrm{mg} / \mathrm{kg}$ EGCG). Dexamethasone and EGCG were administered as appropriate, in saline once daily by intraperitoneal injection.

\section{Animal studies}

Cecal ligation and puncture (CLP) was performed as described previously, but with slight modifications [12]. Pentobarbital (30 $\mathrm{mg} / \mathrm{kg}$ ) was administered intraperitoneally to anesthetize the rats. Using sterile techniques, a $3-\mathrm{cm}$ long incision was made along the midline of the abdomen to allow exposure of the cecum with the adjoining intestine. The cecum was ligated with no bowel obstruction and punctured once with an 18-gauge needle. The cecum was then gently squeezed to leak some fecal material, after which the cecum was returned to the abdominal cavity. In normal control rats, cecum was moved but was not ligated or punctured. Finally, the abdominal incision was closed under sterile conditions. After the operations, all rats were given $3 \mathrm{~mL} / 100 \mathrm{~g}$ body weight of saline solution and antibiotic therapy. All animals were maintained under the same conditions after the surgery.

\section{Specimen collection}

Animals were sacrificed at 6,12 and $24 \mathrm{~h}$ following the surgery. Blood samples and liver tissues were also obtained from rats with CLP and normal control rats. After standing, the serum was separated by centrifugation and frozen at $-80^{\circ} \mathrm{C}$ until analysis. Liver tissues were rapidly removed and frozen at $-80{ }^{\circ} \mathrm{C}$ until subsequent analysis.

\section{Determination of hepatic marker enzymes}

Serum ALT and AST levels were evaluated using commercially available diagnostic kits (Sigma, St. Louis, USA).

\section{Measurement of oxidative stress parameters}

Liver tissue samples were collected at different time-points after CLP, and homogenized separately. The level of MDA in the tissue homogenate was measured according to the method of Ohkawa [13]. Determination of GSH was performed according to the method of Ellman [14]. The SOD activity in the liver tissue was measured using the method described previously [15]. 


\section{Enzyme-linked immunosorbent assay (ELISA)}

Levels of TNF- $\alpha$ and IL-10 were assayed using appropriate commercial kits (Abcam, Cambridge, UK) in line with the manufacturer's instructions.

\section{Quantitative real-time polymerase chain reaction}

TRIzol was used to extract total RNA from rat livers. The extracted total RNA was reversetranscribed with Quantitate Reverse Transcription Kit (Qiagen,Hilden, Germany). Quantitative real-time PCR was performed using SYBR green mix (TOYOBO, Osaka, Japan) on Bio-Rad Real-time PCR platform. The following primer sequences were used:

5'-GCTATTTTCCATTCCCGAGTTAC-3'and 5'ATTGCTGTCCATCTCTGTCAG-3' (for rat Nrf2); 5'-CTTTCAGAAGGGTCAGGTGTC-3' and 5'TGCTTGTTTCGCTCTATCTCC-3' (for rat HO-1); and 5'-GTATTGGGCGCCTGGTCACC-3' and 5'CGCTCCTGGAAGATGGTGATGG-3' (for rat GAPDH).

\section{Western blot analysis}

Western blotting was performed as previously reported [16] using mouse anti-rat Nrf2 (1/1000) monoclonal antibody (Abcam, Cambridge, UK), mouse anti-rat HO-1 (1/5000) monoclonal antibody (Abcam, Cambridge, UK) and mouse anti-rat GAPDH (1/5000) monoclonal antibody (Abcam, Cambridge, UK). This was followed by secondary peroxidase-conjugated antibody (1/2000) (Zhongshan Golden Bridge Biotechnology, Beijing, China). The immunocomplexes were visualized using enhanced chemiluminescence (Cell Signaling Technology, Beverly, USA).

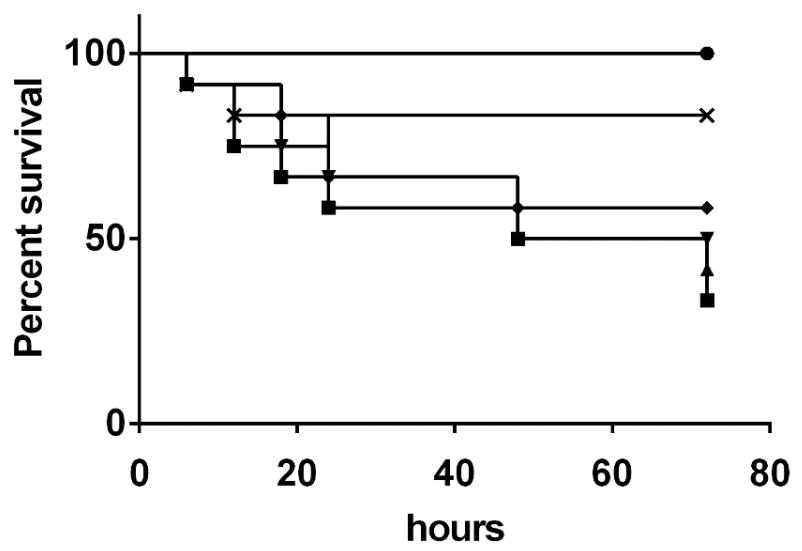

\section{Statistical analysis}

The results are presented as mean \pm standard deviation (SD) and were analyzed using MannWhitney $U$ test. Statistical significance was calculated by Prism 5 software (GraphPad). $P<$ 0.05 were considered significant.

\section{RESULTS}

\section{EGCG treatment improved survival after CLP- induced sepsis.}

The survival of the rats at different time-points following surgery are shown in Figure 1. Survival of normal control rats was $100 \%$ (12/12), whereas the survival of untreated septic rats decreased to $33.3 \%(4 / 12)$ at $72 \mathrm{~h}$ after CLP. Dexamethasone treatment had no significant effects on survival rate when compared with untreated rats after CLP $(p>0.05)$. However, the survival rates of high dose EGCG-treated septic rats were significantly higher than that of the untreated sepsis group and the dexamethasone group $(p<0.05)$.

\section{EGCG protected the rats against sepsis-} induced liver injury

Next, the effects of EGCG treatment on liver injury caused by sepsis were evaluated. Serum samples were collected separately at 6,12 and $24 \mathrm{~h}$ following CLP and used to analyze liver function. It was found that sepsis caused severe liver injury as reflected in increased serum levels of ALT and AST at $6 \mathrm{~h}(p<0.05)$; and at 12 and $24 \mathrm{~h}$ after CLP-induced sepsis $(p<0.01$; Figure 2). Treatment with EGCG, especially high dose EGCG, but not dexamethasone, significantly $(p<$ 0.05 ) decreased serum levels of ALT (Figure 2A) and AST (Figure 2B) after CLP-induced sepsis.

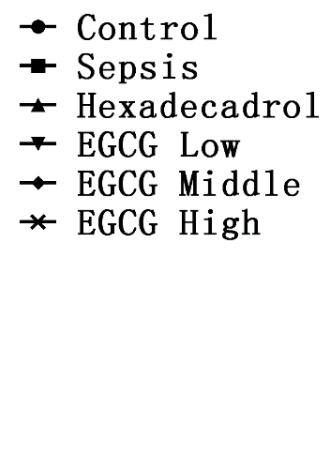

Figure 1: Comparison of the survival rates at $0,6,12,18,24,36,48$ and $72 \mathrm{~h}$ among the six experimental groups after CLP. Data are expressed as percentages 


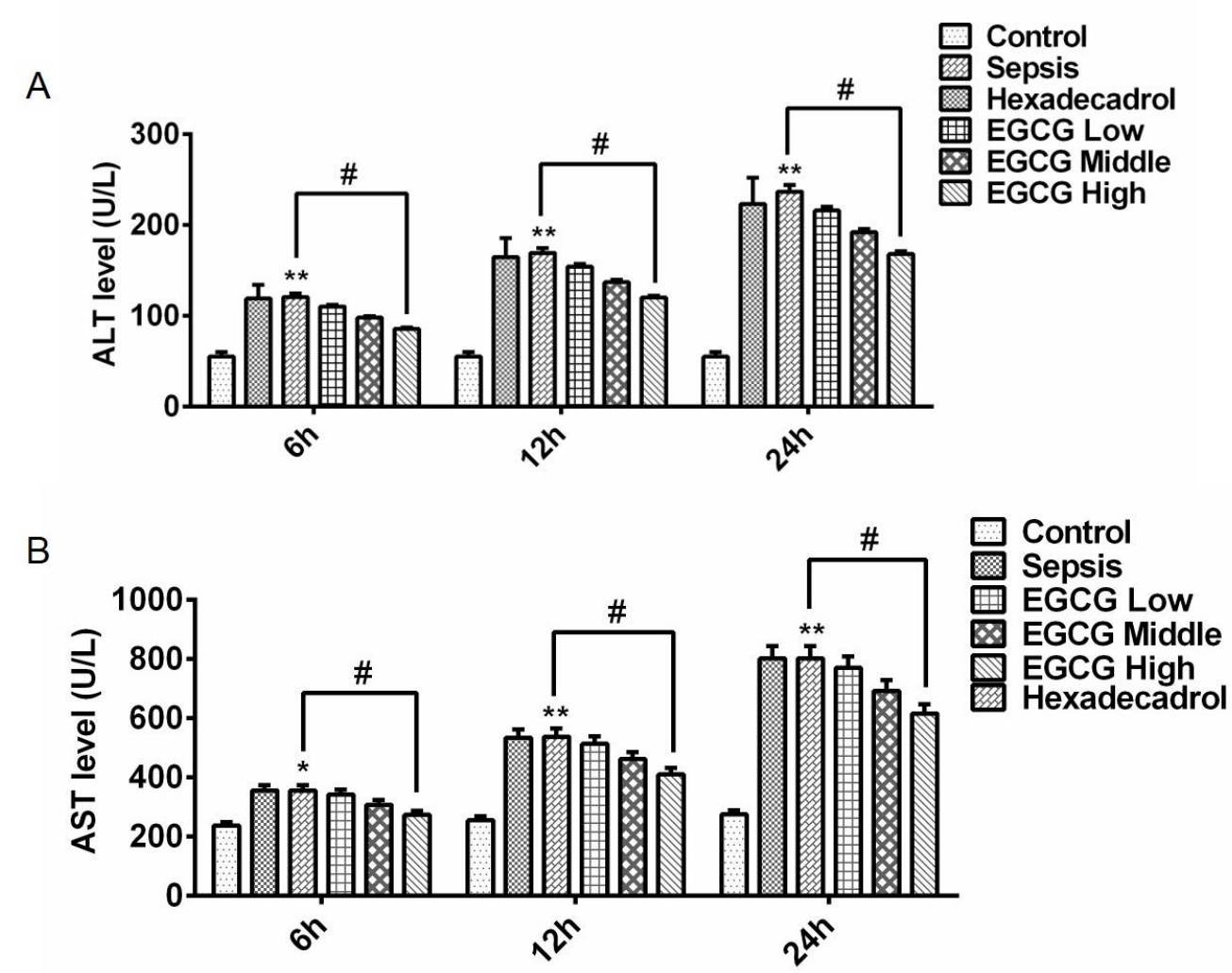

Figure 2: Effect of EGCG on sepsis-induced liver function alterations. ALT levels (A) and AST levels (B) in serum were quantified. Data are presented as mean $\pm S D, n=5$. $\# p<0.05$, when compared with untreated sepsis group; ${ }^{\star} p<0.05$, when compared with normal control group; ${ }^{\star *} p<0.01$, when compared with normal control group

\section{EGCG treatment attenuated oxidative stress during sepsis}

The next step was to analyze some oxidative stress parameters in the liver tissues. The levels of intrahepatic MDA in septic rats were significantly higher after CLP than corresponding values in normal control rats at $6 \mathrm{~h}(p<0.05), 12$ h $(p<0.01)$, and 24 h $(p<0.01)$. The EGCG treatment, however, effectively down-regulated the MDA levels (especially high dose EGCG treatment $(p<0.05)$ after CLP, when compared with untreated sepsis group (Figure 3A). Data from antioxidants analysis showed that the intrahepatic SOD activity and GSH levels were highest in the normal control group, but significantly lower in the untreated sepsis group and dexamethasone-treated sepsis group $(p<$ 0.05 or $p<0.01$ ). However, intrahepatic SOD activity and GSH levels increased significantly after treatment with high dose EGCG $(p<0.05)$. The effects of EGCG on these oxidative stress parameters in the liver were dose-dependent.

\section{EGCG inhibited inflammation due to CLP- induced sepsis}

To test whether EGCG treatment might mitigate inflammation after CLP-induced sepsis, serum levels of TNF- $\alpha$ and IL-10 were assayed. The level of the pro-inflammatory mediator TNF- $\alpha$ was higher in untreated sepsis group than in the normal control group $(p<0.05)$, whereas the anti-inflammatory mediator IL-10 was lower in untreated sepsis group than in the normal control group $(p<0.05)$. Furthermore, high dose EGCG treatment significantly inhibited the CLP-induced inflammation as indicated by decreased production of TNF- $\alpha(p<0.01)$ and increased production of IL-10 $(p<0.01)$ at 12 and $24 \mathrm{~h}$ following CLP (Table 1).

\section{EGCG treatment up-regulate the expression of antioxidant genes}

Intrahepatic Nrf2 and $\mathrm{HO}-1$ expressions as reflected by mRNA and protein levels were decreased significantly $(p<0.01)$ in septic rats when compared with normal control rats. However, their expressions were significantly $(p$ $<0.05$ ) increased after high dose EGCG treatment (Figure 4).

\section{DISCUSSION}

Rat models of CLP-induced sepsis provide useful tools for testing the effect of potential therapeutic agents [17]. Results obtained in the present 
study showed that the plant polyphenol EGCG extracted from Green Tea modulated inflammation, attenuated liver injury and improved survival rate in the CLP-induced sepsis rat model through its anti-oxidant effect.

Previous studies have revealed that liver injury was accompanied by sepsis since increased levels of hepatic enzymes (ALT and AST) which are indicators of impaired hepatic membranes, were always seen during sepsis [18]. Increased serum ALT and AST levels were consistently observed in the CLP-induced septic rats in this study. EGCG treatment at $2 \mathrm{~h}$ after CLP resulted in decreased serum ALT and AST levels, indicating its ability to protect liver functional integrity against sepsis. It would be interesting to carry out further studies on the protective effect of EGCG against other organ injuries during sepsis and other inflammatory diseases.

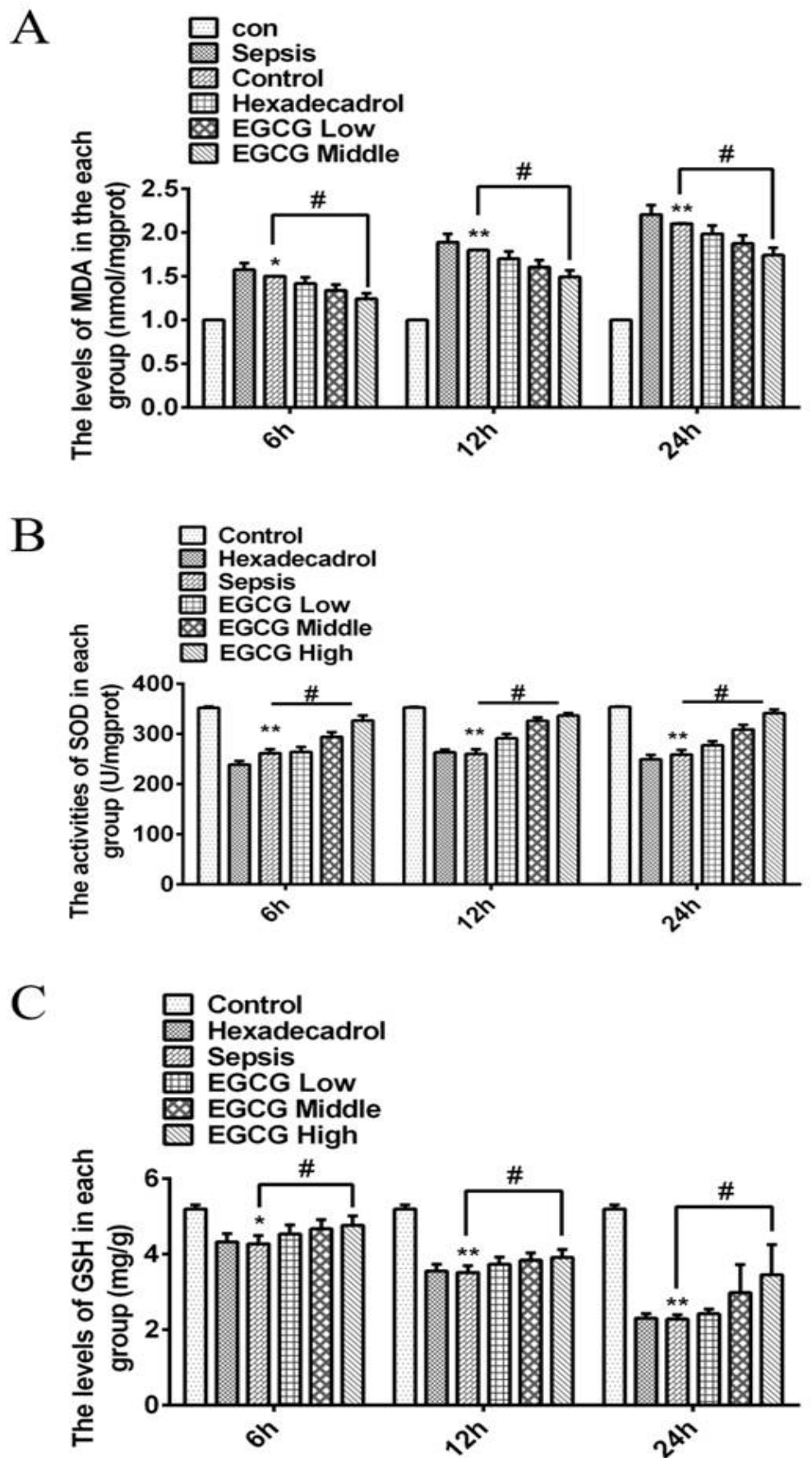

Figure 3: Effect of EGCG on oxidative stress in septic rats. Liver tissue samples were taken at indicated timepoints for measurement of MDA (A), SOD (B) and GSH (C). Data are presented as mean \pm SD, $n=5 ; \# p<0.05$, when compared with untreated sepsis group; ${ }^{*} p<0.05$, when compared with normal control group; ${ }^{* *} p<0.01$, when compared with normal control group 
Table 1: Serum levels of TNF- $\alpha$ and IL-10 in normal control and septic rats at indicated time-points

\begin{tabular}{|c|c|c|c|c|c|c|c|}
\hline \multicolumn{2}{|c|}{ Variable } & Control & Sepsis & Hexadecadrol & $\begin{array}{c}\text { EGCG } \\
\text { Low }\end{array}$ & EGCG Middle & EGCG High \\
\hline \multirow{2}{*}{$6 h$} & $\begin{array}{l}\text { TNF- } \alpha \\
\text { (ng/ml) }\end{array}$ & $44.9 \pm 9.9$ & $48.4 \pm 7.5^{\star}$ & $46.1 \pm 6.8$ & $45.3 \pm 7.7$ & $49.5 \pm 5.8$ & $47.9 \pm 8.8$ \\
\hline & $\begin{array}{c}\mathrm{IL}-10 \\
(\mathrm{ng} / \mathrm{ml})\end{array}$ & $146.9 \pm 18.8$ & $122.8 \pm 13.2^{* *}$ & $121.1 \pm 15.5$ & $126.9 \pm 9.9$ & $121.4 \pm 10.2$ & $122.3 \pm 15.5$ \\
\hline \multirow{2}{*}{$12 \mathrm{~h}$} & $\begin{array}{l}\text { TNF- } \alpha \\
(\mathrm{ng} / \mathrm{ml})\end{array}$ & $45.7 \pm 5.8$ & $53.7 .9 \pm 10.1^{* *}$ & $54.9 \pm 9.11$ & $51.7 \pm 6.12$ & 49.6.9 $99.13 \# \#$ & $47.9 \pm 8.14 \# \#$ \\
\hline & $\begin{array}{c}\mathrm{IL}-10 \\
(\mathrm{ng} / \mathrm{ml})\end{array}$ & $155.1 \pm 9.8$ & $126.8 \pm 13.2^{\star *}$ & $127.1 \pm 15.5$ & $129.9 \pm 5.5$ & $131.4 \pm 13.3 \#$ & $138.2 \pm 11.1 \# \#$ \\
\hline \multirow{2}{*}{$24 h$} & $\begin{array}{l}\text { TNF-a } \\
\text { (ng/ml) }\end{array}$ & $43.1 \pm 4.8$ & $102.3 \pm 7.7^{* *}$ & $99.9 \pm 9.1$ & $80.9 \pm 6.8 \# \#$ & $76.6 \pm 7.5 \# \#$ & $69.9 \pm 8.3 \# \#$ \\
\hline & $\begin{array}{c}\mathrm{IL}-10 \\
(\mathrm{ng} / \mathrm{ml})\end{array}$ & $153.1 \pm 10.3$ & $125.2 \pm 9.4^{\star *}$ & $121.1 \pm 13.8$ & $129.9 \pm 9.9$ & $133.4 \pm 11.2 \#$ & 141.1士13.7\#\# \\
\hline
\end{tabular}

Values are mean $\pm \mathrm{SD} ;{ }^{*} p<0.05$, when compared with normal control group; ${ }^{\star *} p<0.01$, when compared with normal control group. $\# p<0.05$, when compared with untreated sepsis group; \#\#p $<0.01$, when compared with untreated sepsis group

A

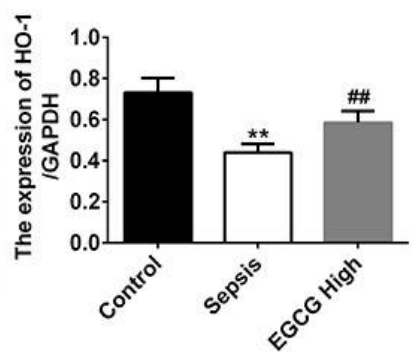

$\mathrm{C}$

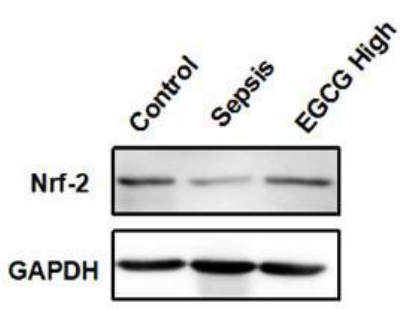

$\mathrm{E}$

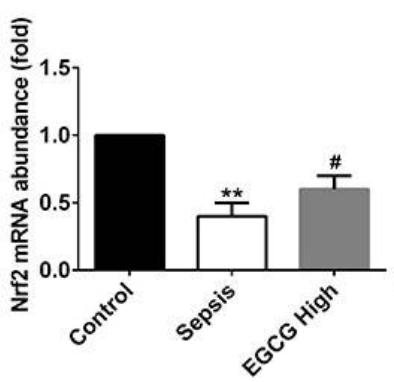

B

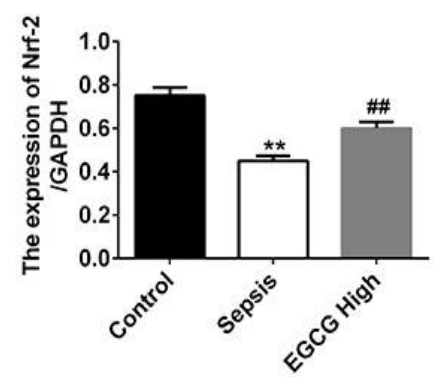

$\mathrm{D}$

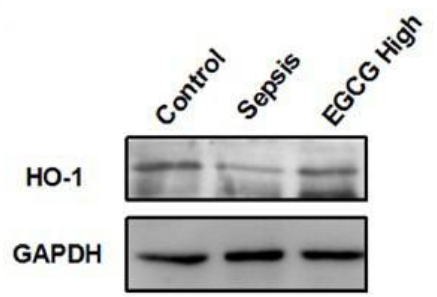

$\mathrm{F}$

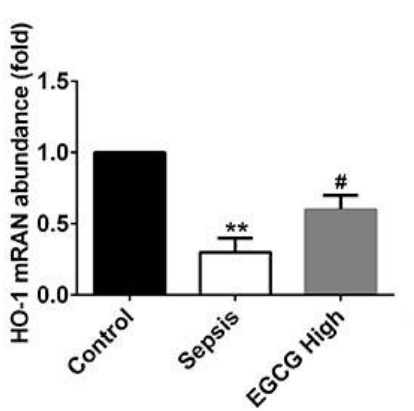

Figure 4: Expression levels of Nrf2 and HO-1 in liver tissues showing mRNA expressions of Nrf2 (A) and HO-1 (B. Nrf2 (C) and HO-1 (D). Data are presented as mean $\pm \mathrm{SD}, \mathrm{n}=4$. ${ }^{* *} p<0.01$, when compared with normal control group; $\# p<0.05$, when compared with untreated sepsis group; \#\# $<0.01$, when compared with untreated sepsis group

Systematic inflammation response is considered a hallmark of sepsis. Among the numerous proand anti-inflammatory cytokines studied during sepsis, TNF- $\alpha$ and IL-10 are of crucial importance [19]. In this study, it was demonstrated that EGCG treatment relieved the inflammation during sepsis. This was demonstrated by the observed significant decrease in TNF- $\alpha$, and significant increase in IL10 levels at different time-points after CLP. 
These results suggest that EGCG may be a potential therapeutic approach to sepsis.

Previous investigations have established that oxidative stress, which is the consequence of imbalance between the levels of cellular oxidants and antioxidants, participates in the pathogenesis of sepsis [9]. Besides, several studies have shown the effect of anti-oxidant drugs on improvement of survival during sepsis [20-22].

Polyphenols are secondary metabolites seen largely in plants. They have attracted much attention for their potential health benefits because of their antioxidant properties and safety [23]. EGCG is the major catechin in green tea and is considered an antioxidant which prevents oxidative stress. In animal models, EGCG exhibits protective roles in varied pathological conditions, including Parkinson's disease and diabetes [23-25].

In the present study, it has been shown that EGCG reduced sepsis-induced oxidative stress through its effect on the levels of hepatic oxidative stress parameters such as SOD and GSH. Furthermore, EGCG promoted the expressions of hepatic Nrf2 and $\mathrm{HO}-1$, indicating the implication of Nrf2/HO-1 signaling pathway in the protective effect of EGCG against sepsis.

\section{CONCLUSION}

The results of this study suggest that EGCG has a potential for use as a treatment strategy for sepsis and sepsis-induced liver injury as demonstrated by its anti-oxidant and antiinflammatory properties.

\section{DECLARATIONS}

\section{Conflict of interest}

No conflict of interest is associated with this work.

\section{Contributions of authors}

The authors declare that this work was done by the authors named in this article and all liabilities pertaining to claims relating to the content of this article will be borne by them. Jian-xin Yang wrote this paper and participated in the study design and experiments, $\mathrm{Yu}$-lin $\mathrm{Li}$ performed the experiments and analyzed the data, Ning-chuan Shi designed this study and supervised the experiments.

\section{REFERENCES}

1. Wang HE, Shapiro NI, Angus DC, Yealy DM. National estimates of severe sepsis in United States emergency departments. Crit Care Med 2007; 35: 1928-1936.

2. Riedemann NC, Guo RF, Ward PA. Novel strategies for the treatment of sepsis. Nat Med 2003; 9: 517-524.

3. Bauer M, Press AT, Trauner M. The liver in sepsis: patterns of response and injury. Curr Opin Crit Care 2013; 19: 123-127.

4. Yan J, Li S, Li S. The role of the liver in sepsis. Int Rev Immunol 2014; 33: 498-510.

5. Kramer $L$, Jordan B, Druml W, Bauer $P$, Metnitz PG, Austrian Epidemiologic Study on Intensive Care ASG. Incidence and prognosis of early hepatic dysfunction in critically ill patients--a prospective multicenter study. Crit Care Med 2007; 35: 1099-1104.

6. Jones DP. Radical-free biology of oxidative stress. Am J Physiol Cell Physiol 2008; 295: C849-C868.

7. Macdonald J, Galley HF, Webster NR. Oxidative stress and gene expression in sepsis. Br J Anaesth 2003; 90: 221-232.

8. Li S, Hong M, Tan HY, Wang N, Feng $Y$. Insights into the Role and Interdependence of Oxidative Stress and Inflammation in Liver Diseases. Oxid Med Cell Longev 2016; 2016: 4234061.

9. Prauchner CA. Oxidative stress in sepsis: Pathophysiological implications justifying antioxidant cotherapy. Burns 2017; 43: 471-485.

10. Beecher GR, Warden BA, Merken H. Analysis of tea polyphenols. Proc Soc Exp Biol Med 1999; 220: 267270

11. U.S. National Institutes of Health. Laboratory animal welfare: Public Health Service policy on humane care and use of laboratory animals by awardee institutions; notice. Fed Regist, 1985, 50(90):19584.

12. Barichello T, Martins MR, Reinke A, Feier G, Ritter C, Quevedo J, Dal-Pizzol F. Cognitive impairment in sepsis survivors from cecal ligation and perforation. Crit Care Med 2005; 33: 221-3; discussion 262-263.

13. Ohkawa H, Ohishi N, Yagi K. Assay for lipid peroxides in animal tissues by thiobarbituric acid reaction. Anal Biochem 1979; 95: 351-358.

14. Ellman GL. Tissue sulfhydryl groups. Arch Biochem Biophys 1959; 82: 70-77.

15. Marklund S, Marklund G. Involvement of the superoxide anion radical in the autoxidation of pyrogallol and a convenient assay for superoxide dismutase. Eur $J$ Biochem 1974; 47: 469-474.

16. Li H, Song F, Duan LR, Sheng JJ, Xie YH, Yang Q, Chen $Y$, Dong $Q Q$, Zhang BL, Wang SW. Paeonol and danshensu combination attenuates apoptosis in myocardial infarcted rats by inhibiting oxidative stress: Roles of Nrf2/HO-1 and PI3K/Akt pathway. Sci Rep 2016; 6: 23693.

17. Hubbard WJ, Choudhry M, Schwacha MG, Kerby JD, Rue LW, 3rd, Bland KI, Chaudry IH. Cecal ligation and puncture. Shock 2005; 24 Suppl 1: 52-57. 
18. Makled MN, El-Awady MS, Abdelaziz RR, Atwan N, Guns ET, Gameil NM, Shehab El-Din AB, Ammar EM. Pomegranate protects liver against cecal ligation and puncture-induced oxidative stress and inflammation in rats through TLR4/NF-kappaB pathway inhibition. Environ Toxicol Pharmacol 2016; 43: 182-192.

19. Chousterman BG, Swirski FK, Weber GF. Cytokine storm and sepsis disease pathogenesis. Semin Immunopathol 2017; 39: 517-528.

20. Barichello T, Machado RA, Constantino L, Valvassori SS, Reus GZ, Martins MR, Petronilho F, Ritter C, Quevedo J, Dal-Pizzol F. Antioxidant treatment prevented late memory impairment in an animal model of sepsis. Crit Care Med 2007; 35: 2186-2190.

21. Ritter C, Andrades ME, Reinke A, Menna-Barreto S, Moreira JC, Dal-Pizzol F. Treatment with $\mathrm{N}$ acetylcysteine plus deferoxamine protects rats against oxidative stress and improves survival in sepsis. Crit Care Med 2004; 32: 342-349.

22. Zheng $Y$, Zhu D. Molecular Hydrogen Therapy Ameliorates Organ Damage Induced by Sepsis. Oxid Med Cell Longev 2016; 2016: 5806057.

23. Pandey KB1, Rizvi SI. Plant polyphenols as dietary antioxidants in human health and disease. Oxid Med Cell Longev 2009; 2: 270-278.

24. Yan J, Zhao Y, Suo S, Liu Y, Zhao B. Green tea catechins ameliorate adipose insulin resistance by improving oxidative stress. Free Radic Biol Med 2012; 52: 1648-1657.

25. Orsolic N, Sirovina D, Gajski G, Garaj-Vrhovac V, Jazvinscak Jembrek M, Kosalec I. Assessment of DNA damage and lipid peroxidation in diabetic mice: effects of propolis and epigallocatechin gallate (EGCG). Mutat Res 2013; 757: 36-44. 